\title{
A NEW PARAdigM FOR HAPTIC RENDERING BASED ON ORTHOGONAL DECOMPOSITION
}

\author{
Sepulveda Cervantes, G.; Dominguez Ramirez, O.A. \& \\ PARRA VEGA, V.
}

Abstract: A new paradigm for simultaneously haptic rendering of dynamic and surface properties for virtual objects based on orthogonal decomposition is presented. The methodology is used to recreate lineal dynamics, stiffness and elasticity and surface properties like tangent friction for virtual objects defined by differentiable manifolds. A stability analysis of the energetic coupling between the haptic device, virtual object and human user interaction using Lyapunov function is presented. The methodology is tested using two different experimental platforms, the first with an advanced haptic device and using a real time operating system, the second platform uses both commercial haptic device and commercial operating system. Experimental results showing a stable interaction in both platforms are presented.

Key words: Haptic rendering, virtual reality, nonlineal control, robotics
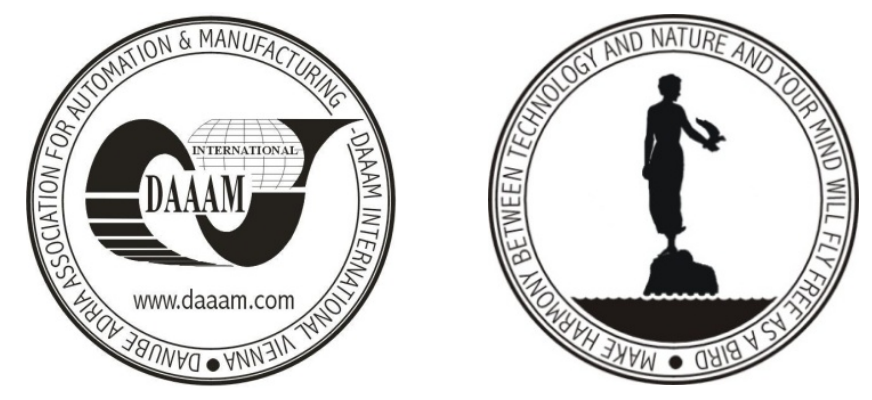

Authors' data: B.D. PhD. Student Sepulveda Cervantes, G[abriel]*; Prof. PhD. Dominguez Ramirez, O[mar] A[rturo]**, M.Sc. Prof. PhD. Parra Vega, V[icente]***, *Ocoyoacac 44 Col. Cumbria Cuautitlán Izcalli Estado de México, CP 54740, México, **San Francisco No. 140, Fraccionamiento El Carmen, Pachuca, Hidalgo, zip 42030, Mexico, ***Robotics and Advanced Manufacturing Division Research Center for Advanced Studies Saltillo Campus - CINVESTAV Carretera Saltillo-Monterrey Km 13.5, Ramos Arizpe, Coah, 25900, México, gsepulveda@cinvestav.mx, omar@uaeh.reduaeh.mx, vparra@cinvestav.mx

This Publication has to be referred as: Sepulveda Cervantes, G[abriel]; Dominguez Ramirez, O[mar] A[rturo] \& Parra Vega, V[icente] (2008). A New Paradigm for Haptic Rendering Based on Orthogonal Decomposition, Chapter 60 in DAAAM International Scientific Book 2008, pp. 743-758, B. Katalinic (Ed.), Published by DAAAM International, ISBN 978-3-901509-66-7, ISSN 1726-9687, Vienna, Austria

DOI: $10.2507 /$ daaam.scibook.2008.60 